\title{
Simple synthesis of
} pyrrolo[3,2-e]indole-1-carbonitriles

\author{
Adam Trawczyński ${ }^{1,2}$, Robert Bujok ${ }^{1}$, Zbigniew Wróbel ${ }^{1}$ \\ and Krzysztof Wojciechowski ${ }^{* 1}$
}

Open Access

\author{
Full Research Paper \\ Address: \\ ${ }^{1}$ Institute of Organic Chemistry, Polish Academy of Sciences, ul. \\ Kasprzaka 44/52, POBox 58, 01-224 Warszawa, Poland, Fax: +48 \\ (22) 6326681 and ${ }^{2}$ Department of Chemistry, Warsaw University of \\ Technology, ul. Noakowskiego 3, 00-664 Warszawa, Poland \\ Email: \\ Krzysztof Wojciechowski* - krzysztof.wojciechowski@icho.edu.pl \\ * Corresponding author \\ Keywords: \\ alkylation; ketones; nitriles; pyrroloindole; reduction; \\ trimethylchlorosilane
}

Beilstein J. Org. Chem. 2013, 9, 934-941. doi:10.3762/bjoc. 9.107

Received: 10 March 2013

Accepted: 23 April 2013

Published: 15 May 2013

Associate Editor: J. P. Wolfe

(c) 2013 Trawczyński et al; licensee Beilstein-Institut. License and terms: see end of document.

\begin{abstract}
Alkylation of 5-nitroindol-4-ylacetonitriles with ethyl chloroacetate, $\alpha$-halomethyl ketones, and chloroacetonitrile followed by a treatment of the products with chlorotrimethylsilane in the presence of DBU gives 1-cyanopyrrolo[3,2-e]indoles substituted in position 2 with electron-withdrawing groups.
\end{abstract}

\section{Introduction}

Indole and its analogues bearing condensed arene and heteroarene rings are privileged structures amongst biologically active compounds. The 1,2-dihydropyrrolo[3,2-e]indole fragment is present in anticancer agents, such as CC-1065 [1], duocarmycin [1], and yatakemycin [2]. Some pyrrolo[3,2$e$ ]indole derivatives show antimicrobial activity [3]. One method of synthesis of the 1,2-dihydropyrrolo[2,3-e]indoles is reduction of pyrrolo[3,2-e]indoles with sodium cyanoborohydride [4]. On the other hand there are many methods of synthesis of pyrrolo[3,2-e]indoles such as the copper-catalyzed transformation of tetrahydroquinoline derivatives [4], photochemical cyclization of 1,2-bis(2-pyrrolo)ethylenes [5], the Fischer indole synthesis from indol-5-ylhydrazones [3], or a palladium-catalyzed hydrogenation of 5-nitroindol-4-ylaceto- nitriles 2 [6]. In the latter synthesis of pyrrolo[3,2-e]indole 3 the starting nitrile 2 was obtained by the vicarious nucleophilic substitution (VNS) [7-11] of hydrogen in 1-alkyl-5-nitroindole 1 with 4-chlorophenoxyacetonitrile [12] (Scheme 1).

In our previous papers [13-16] we have shown that $o$-nitroarylacetonitriles alkylated and alkenylated at the $\alpha$-position to the cyano group can be converted into indoles under basic conditions in the presence of a silylating agent.

\section{Results and Discussion}

Here we report a simple two-step procedure for the transformation of 5-nitroindol-4-ylacetonitriles into pyrrolo[3,2-e]indole1 -carbonitriles 6 bearing an additional electron-withdrawing 
<smiles>[R]n1ccc2cc([N+](=O)[O-])ccc21</smiles>

1

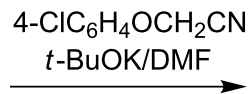

ref. [12]<smiles>[R]n1ccc2c(CC#N)c([N+](=O)[O-])ccc21</smiles>

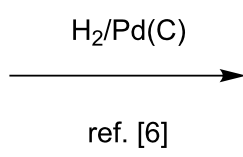

Scheme 1: Synthesis of pyrrolo[3,2-e]indoles via VNS in 5-nitroindoles [6,12].

substituent at position 2 . In our approach the starting material was 1-benzyloxymethyl-4-cyanomethyl-2-methyl-5-nitroindole (4) obtained via the VNS of hydrogen in 1-benzyloxymethyl-2methyl-5-nitroindole with 4-chlorophenoxyacetonitrile according to our earlier elaborated method [12]. Alkylation of the nitrile 4 with ethyl bromoacetate in the presence of $\mathrm{K}_{2} \mathrm{CO}_{3}$ led to the expected cyanoester $\mathbf{5 a}$ in $68 \%$ yield, but the product contained some contaminants difficult to separate by crystallization or column chromatography. Searching for more convenient reaction conditions, we have found that this reaction proceeds satisfactorily in almost quantitative yield when diazabicycloundecene (DBU) was used as the base. Analogous alkylation with $\alpha$-halomethyl ketones, chloroacetonitrile, chloroacetamide and cinnamyl bromide provided the expected alkylation products $\mathbf{5 b}-\mathbf{g}$ in good yields (Scheme 2 and Table 1).
To find optimal conditions for cyclization of the model compound 5a we screened various combinations of base and a reagent promoting the cyclization. With chlorotrimethylsilane-triethylamine the reaction proceeded slowly, and the starting material was completely consumed after $24 \mathrm{~h}$, but the product $6 \mathbf{6}$ was isolated in moderate $30 \%$ yield. However, when we replaced triethylamine with a stronger base, such as DBU, the reaction was completed in $30 \mathrm{~min}$, and the product was isolated in $90 \%$ yield. Similarly, with $N, O$ bis(trimethylsilyl)acetamide (BSA) the reaction was completed in 30 min giving 6 a in $67 \%$ yield. With tributylchlorostannane combined with DBU the reaction proceeded slowly to form after $24 \mathrm{~h}$ product $\mathbf{6 a}$ in $72 \%$ yield. Methanesulfonyl and pivaloyl chlorides, in combination with DBU proved ineffective in this reaction giving a very low rate of conversion after 24 h. Thus, transformations of other nitriles $\mathbf{5} \mathbf{b}-\mathbf{g}$ into pyrrolo[3,2-

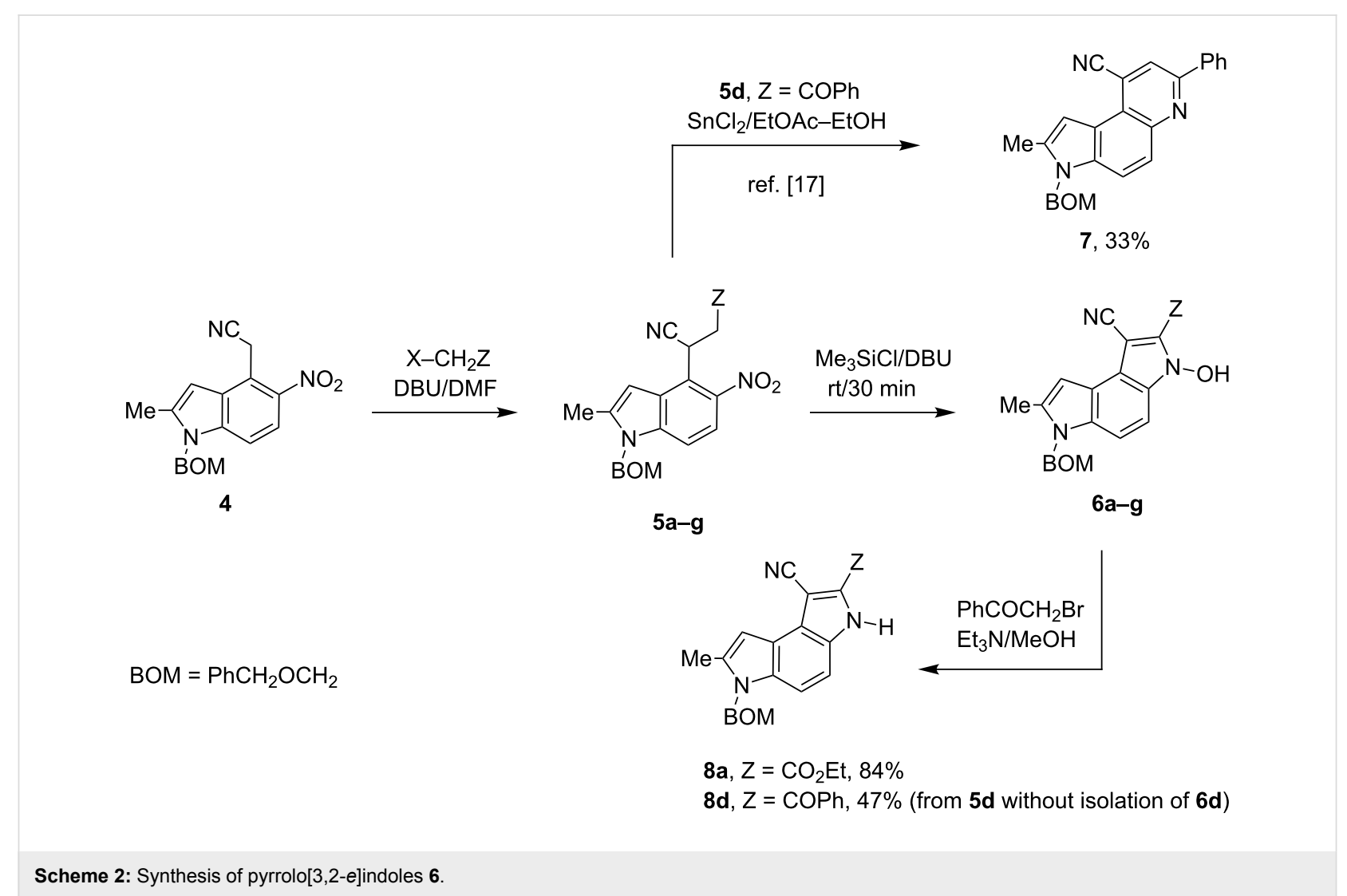


Table 1: Alkylation products 5 and synthesized 1-cyano-3-hydroxy-pyrrolo[3,2-e]indoles 6.

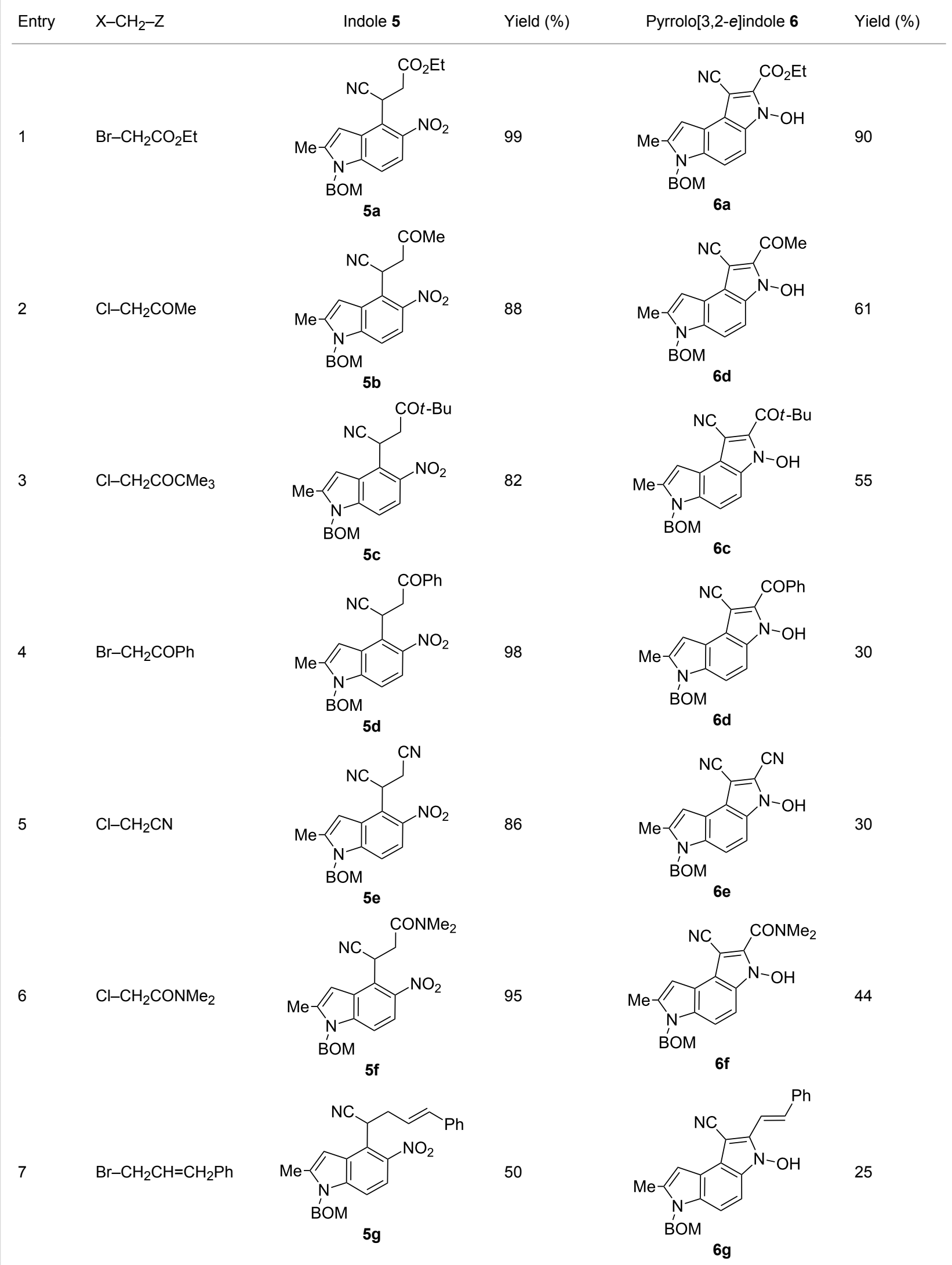


e]indoles 6 were performed in the DBU-chlorotrimethylsilane system, and the results are presented in Table 1. It is worth mentioning that the ketone 5d upon reduction with $\mathrm{SnCl}_{2}$ cyclized to pyrrolo[3,2-f]quinoline-9-carbonitrile 7 [17].

The removal of the benzyloxymethyl group from 1-(benzyloxymethyl)pyrrolo[3,2-e]indoles by catalytic hydrogenation has been described by Macor [6]. The hydroxy group from the $N$-hydroxypyrrole fragment can be removed by a procedure elaborated by us [18] employing $\alpha$-bromoacetophenone in the presence of triethylamine as exemplified for pyrroloindoles $\mathbf{6 a}$ and $\mathbf{6 d}$ that were transformed under these conditions into the corresponding derivatives $8 \mathbf{a}$ and $\mathbf{8 d}$ (Scheme 2). The crude 3-hydroxy-pyrrolo[3,2-e]indole $\mathbf{6 d}$ without isolation and purification was subjected to dehydroxylation giving compound $\mathbf{8 d}$ in $47 \%$ yield.

A plausible route to the formation of 3-hydroxy-1-cyanopyrrolo[3,2-e]indoles is exemplified by the synthesis of 1,2dicyano derivative $6 \mathbf{e}$ from the dinitrile $5 \mathbf{e}$ (Scheme 3 ). In the first step the $o$-nitrobenzylic carbanion is silylated with trimethylchlorosilane to form trimethylsilyl nitronate $\mathbf{A}$. Then a consecutive deprotonation forms another carbanion $\mathbf{B}$ at the $\beta$-position to the ring. The attack of this carbanion on the trimethylsilylnitronate results in the substitution of trimethoxysiloxyl and formation of $\mathbf{C}$ in that the fivemembered ring finally isomerizes to the $N$-hydroxypyrrole fragment of $\mathbf{6 e}$.

To remove the benzyloxymethyl group from the compound $\mathbf{8 a}$ we adopted the procedure proposed by Macor [6]. Heating $\mathbf{8 a}$ with ammonium formate and $10 \%$ palladium on carbon as a catalyst in isopropanol in a sealed tube $\left(95^{\circ} \mathrm{C}\right)$ led to a mixture of the expected product 9a and the product 10a in that the cyano group was reduced to a methyl substituent (Scheme 4). There is a literature precedence [19] for similar transformations of cyanoarenes into corresponding methyl derivatives upon transfer hydrogenation with ammonium formate in the presence of palladium on a carbon catalyst.

\section{Conclusion}

In conclusion, the approach presented herein can be useful for the synthesis of variously substituted pyrrolo[3,2-e]indoles. The method does not require reductive conditions for the formation of the pyrrole ring and, thus, can be applicable for derivatives bearing sensitive substituents.<smiles>[R5]n1c(C)cc2c(C(C#N)CC#N)c([N+](=O)[O-])ccc21</smiles>

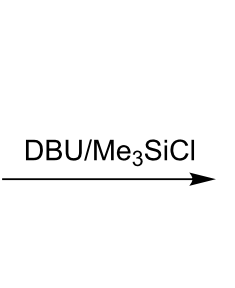<smiles></smiles>

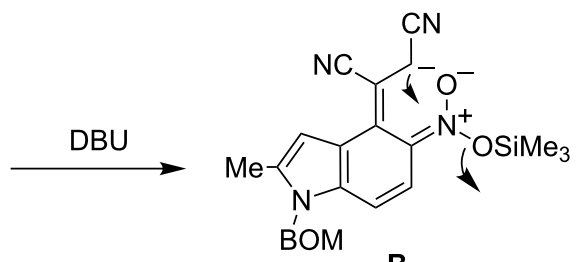

A<smiles>[3H]C([B])C([3H])([O-])[18OH]</smiles><smiles>CC1=Cc2c(C#N)c(C#N)n(O)c2C=CN1C</smiles><smiles>CCCC[Te]</smiles><smiles></smiles>

Scheme 3: Plausible route for transformation of indoles 5 into pyrrolo[3,2-e]indoles 6.<smiles>CCOC(=O)c1[nH]c2ccc3c(cc(C)n3COc3ccccc3)c2c1C#N</smiles>

$8 a$<smiles>CCOC(=O)c1[nH]c2ccc3[nH]c(C)cc3c2c1C#N</smiles>

9a (22\%)<smiles>CCOC(=O)c1[nH]c2ccc3[nH]c(C)cc3c2c1C</smiles>

$10 a(34 \%)$ 


\section{Experimental}

Melting points (mp) are uncorrected. ${ }^{1} \mathrm{H}$ and ${ }^{13} \mathrm{C}$ NMR spectra were recorded on a Bruker Avance 500 or Varian vnmr s500 (both $500 \mathrm{MHz}$ for ${ }^{1} \mathrm{H}$ and $125 \mathrm{MHz}$ for ${ }^{13} \mathrm{C}$ spectra) instruments at $298 \mathrm{~K}$. Chemical shifts $\delta$ are expressed in parts per million referenced to TMS; coupling constants $J$ in hertz. IR spectra were recorded in $\mathrm{KBr}$ on a Perkin Elmer PE Spectrum 2000 spectrometer. Electron impact mass spectra (EI, $70 \mathrm{eV}$ ) were obtained on AMD-604 and AutoSpec Premier spectrometer. Electrospray mass spectra (ESI) were obtained on 4000 Q-TRAP and SYNAPT G2-S HDMS. Silica gel (Merck 60, 230-400 mesh) was used for column chromatography (CC). All reagents and solvents were of reagent grade or purified according to standard methods before use. 1-Benzyloxymethyl4-(cyanomethyl)-2-methyl-5-nitroindole (4) was obtained by VNS of hydrogen in 1-benzyloxymethyl-2-methyl-5-nitroindole with 4-chlorophenoxyacetonitrile following our previously elaborated method [12].

\section{Alkylation of indolylacetonitrile 4 with ethyl bromo- acetate. Synthesis of 3-(1-benzyloxymethyl-2- methyl-5-nitro- $1 \mathrm{H}$-indol-4-yl)-3-cyanopropionic acid ethyl ester (5a) - Typical procedure}

A solution of (1-benzyloxymethyl-2-methyl-5-nitro-1H-indol-4yl)acetonitrile $(4,0.335 \mathrm{~g}, 1 \mathrm{mmol})$ and ethyl bromoacetate $(0.25 \mathrm{~g}, 1.5 \mathrm{mmol})$ were stirred in DMF $(5 \mathrm{~mL})$ and DBU $(0.30 \mathrm{~g}, 2 \mathrm{mmol})$ at $60{ }^{\circ} \mathrm{C}$ until the starting material 4 disappeared (usually $24 \mathrm{~h}$, TLC control). Then the reaction mixture was poured into diluted $\mathrm{HCl}$ and the product was extracted with EtOAc $(3 \times 30 \mathrm{~mL})$ and dried with $\mathrm{Na}_{2} \mathrm{SO}_{4}$. After evaporation of the solvent the residue was purified by column chromatography $\left(\mathrm{SiO}_{2}\right.$, hexane-EtOAc, 2:1). Yellow crystals; mp $76-78{ }^{\circ} \mathrm{C} ;{ }^{1} \mathrm{H} \mathrm{NMR}\left(500 \mathrm{MHz}, \mathrm{CDCl}_{3}\right) \delta 1.28(\mathrm{t}, J=7.3 \mathrm{~Hz}$, $3 \mathrm{H}), 2.52(\mathrm{~s}, 3 \mathrm{H}), 3.06(\mathrm{dd}, J=16.9,6.1 \mathrm{~Hz}, 1 \mathrm{H}), 3.28(\mathrm{dd}$, $J=16.9,9.0 \mathrm{~Hz}, 1 \mathrm{H}), 4.21(\mathrm{q}, J=7.3 \mathrm{~Hz}, 2 \mathrm{H}), 4.47(\mathrm{~s}, 2 \mathrm{H})$, $5.42(\mathrm{dd}, J=9.0,6.1 \mathrm{~Hz}, 1 \mathrm{H}), 5.55(\mathrm{~s}, 2 \mathrm{H}), 6.80$ (br s, 1H), 7.23-7.27 (m, 2H), 7.31-7.37 (m, 4H), 7.84 (d, $J=9.0 \mathrm{~Hz}, 1 \mathrm{H})$; ${ }^{13} \mathrm{C}$ NMR $\left(125 \mathrm{MHz}, \mathrm{CDCl}_{3}\right) \delta 12.82,14.10,27.65,37.67$, 61.66, 70.12, 71.96, 102.16, 109.93, 118.79, 119.02, 120.50, $126.92,127.66,128.29,128.63,136.43,139.48,141.32,142.22$, 168.98; IR (KBr, cm $\left.{ }^{-1}\right)$ v: 2979, 2243, 1728, 1603, 1561, 1510, 1461, 1450, 1409, 1373, 1339, 1272, 1244, 1205, 1188, 1130, $1096,959,813,800,769,759,741$; EIMS $(70 \mathrm{eV}) \mathrm{m} / \mathrm{z}$ (\% relative intensity): 421 (14) [M] ${ }^{+\cdot}, 404$ (5), 92 (8), 91 (100); HRMS-EI $(70 \mathrm{eV}, \mathrm{m} / \mathrm{z}):[\mathrm{M}]^{+}$calcd for $\mathrm{C}_{23} \mathrm{H}_{23} \mathrm{~N}_{3} \mathrm{O}_{5}$, 421.1638; found, 421.1630 .

2-(1-Benzyloxymethyl-2-methyl-5-nitro- $1 H$-indol-4-yl)-4oxopentanenitrile (5b). Yellow crystals; mp $102-104{ }^{\circ} \mathrm{C}$; ${ }^{1} \mathrm{H}$ NMR $\left(500 \mathrm{MHz}, \mathrm{CDCl}_{3}\right) \delta 2.21(\mathrm{~s}, 3 \mathrm{H}), 2.52(\mathrm{~d}, J=1.0 \mathrm{~Hz}$, $3 \mathrm{H}), 3.12(\mathrm{dd}, J=18.3,5.2 \mathrm{~Hz}, 1 \mathrm{H}), 3.50(\mathrm{dd}, J=18.3,8.7 \mathrm{~Hz}$,
1H), 4.47 (s, 2H), 5.37 (dd, $J=8.7,5.2 \mathrm{~Hz}, 1 \mathrm{H}), 5.54(\mathrm{~s}, 2 \mathrm{H})$, 6.77 (br s, 1H), 7.24-7.27 (m, 2H), 7.31-7.37 (m, 4H), 7.83 (d, $J=8.9 \mathrm{~Hz}, 1 \mathrm{H}) ;{ }^{13} \mathrm{C} \mathrm{NMR}\left(125 \mathrm{MHz}, \mathrm{CDCl}_{3}\right) \delta 12.80,26.20$, 29.42, 46.23, 70.06, 71.92, 102.12, 109.73, 118.82, 119.21, 121.08, 127.03, 127.64, 128.25, 128.62, 136.39, 139.40, 141.21, 142.19, 202.77; IR (KBr, $\left.\mathrm{cm}^{-1}\right)$ v: 2915, 2250, 2240, 1714, $1607,1559,1517,1504,1451,1400,1330,1257,1238,1170$, 1081, 1060, 1006, 948, 817, 733; EIMS $(70 \mathrm{eV}) \mathrm{m} / \mathrm{z}$ (\% relative intensity): 391 (3) $[\mathrm{M}]^{+}, 357$ (11), 92 (8), 91 (100); HRMS-EI $(70 \mathrm{eV}, \mathrm{m} / \mathrm{z}):[\mathrm{M}]^{+}$calcd for $\mathrm{C}_{22} \mathrm{H}_{21} \mathrm{~N}_{3} \mathrm{O}_{4}$, 391.1532; found, 391.1540 .

2-(1-Benzyloxymethyl-2-methyl-5-nitro-1 H-indol-4-yl)-5,5dimethyl-4-oxohexanenitrile (5c). Yellow crystals; $\mathrm{mp}$ 92-94 ${ }^{\circ} \mathrm{C} ;{ }^{1} \mathrm{H}$ NMR $\left(500 \mathrm{MHz}, \mathrm{CDCl}_{3}\right) \delta 1.15(\mathrm{~s}, 9 \mathrm{H}), 2.53(\mathrm{~d}$, $J=0.8 \mathrm{~Hz}, 3 \mathrm{H}), 3.14(\mathrm{dd}, J=17.9,4.9 \mathrm{~Hz}, 1 \mathrm{H}), 3.57(\mathrm{dd}$, $J=17.9,9.1 \mathrm{~Hz}, 1 \mathrm{H}), 4.47(\mathrm{~s}, 2 \mathrm{H}), 5.32(\mathrm{dd}, J=9.1,4.9 \mathrm{~Hz}$, $1 \mathrm{H}), 5.55$ (s, 2H), 6.78 (br s, 1H), 7.24-7.37 (m, 6H), 7.83 (d, $J$ $=9.0 \mathrm{~Hz}, 1 \mathrm{H}) ;{ }^{13} \mathrm{C} \mathrm{NMR}\left(125 \mathrm{MHz}, \mathrm{CDCl}_{3}\right) \delta 12.84,26.11$, 26.91, 40.21, 44.06, 70.05, 71.92, 102.10, 109.66, 118.83, $119.42,121.47,127.10,127.65,128.26,128.62,136.40,139.37$, 141.18, 142.21, 210.44; IR (KBr, $\left.\mathrm{cm}^{-1}\right)$ v: 2969, 2244, 1707, 1606, 1652, 1519, 1477, 1340, 1071, 1029, 818, 757, 740; EIMS (70 eV) $m / z$ (\% relative intensity): 433 (3) $[\mathrm{M}]^{+\cdot}, 399$ (6), 92 (8), 91 (100); HRMS-EI (70 eV, $m / z):[\mathrm{M}]^{+}$calcd for $\mathrm{C}_{25} \mathrm{H}_{27} \mathrm{~N}_{3} \mathrm{O}_{4}$, 433.2002; found, 433.2004 .

2-(1-Benzyloxymethyl-2-methyl-5-nitro-1 H-indol-4-yl)-4oxo-4-phenylbutyronitrile (5d). Yellow crystals; mp 123-125 ${ }^{\circ} \mathrm{C} ;{ }^{1} \mathrm{H}$ NMR $\left(500 \mathrm{MHz}, \mathrm{CDCl}_{3}\right) \delta 2.52(\mathrm{~d}, J=1.0 \mathrm{~Hz}$, $3 \mathrm{H}), 3.68(\mathrm{dd}, J=18.0,5.1 \mathrm{~Hz}, 1 \mathrm{H}), 4.05(\mathrm{dd}, J=18.0,8.7 \mathrm{~Hz}$, $1 \mathrm{H}), 4.47(\mathrm{~s}, 2 \mathrm{H}), 5.55(\mathrm{~s}, 2 \mathrm{H}), 5.58(\mathrm{dd}, J=8.7,5.1 \mathrm{~Hz}, 1 \mathrm{H})$, $6.83(\mathrm{~m}, 1 \mathrm{H}), 7.25(\mathrm{~s}, 1 \mathrm{H}), 7.26(\mathrm{~s}, 1 \mathrm{H}), 7.30-7.37(\mathrm{~m}, 4 \mathrm{H})$, 7.44-7.48 (m, 2H), 7.56-7.60 (m, 1H), $7.85(\mathrm{~d}, J=9.0 \mathrm{~Hz}, 1 \mathrm{H})$, 7.93-7.96 (m, $2 \mathrm{H}) ;{ }^{13} \mathrm{C} \mathrm{NMR}\left(125 \mathrm{MHz}, \mathrm{CDCl}_{3}\right) \delta 12.86$, 26.64, 42.01, 70.09, 71.96, 102.24, 109.78, 118.88, 119.46, 121.36, 127.15, 127.67, 128.17, 128.27, 128.64, 128.78, 133.85, 135.57, 136.44, 139.45, 141.27, 142.37, 194.46; IR (KBr, cm $\left.{ }^{-1}\right)$ v: 2921, 2246, 1690, 1559, 1518, 1447, 1343, 1213, 1086, 1071, 803, 751, 689; EIMS (70 eV) m/z (\% relative intensity): 453 (2) $[\mathrm{M}]^{+\cdot}, 419$ (9), 105 (35), 92 (8), 91 (100), 77 (13); HRMS-EI (70 eV, m/z): $[\mathrm{M}]^{+}$calcd for $\mathrm{C}_{27} \mathrm{H}_{23} \mathrm{~N}_{3} \mathrm{O}_{4}, 453.1689$; found, 453.1671 .

2-(1-Benzyloxymethyl-2-methyl-5-nitro-1H-indol-4-yl)succinonitrile (5e). Brown oil; ${ }^{1} \mathrm{H}$ NMR $\left(500 \mathrm{MHz}, \mathrm{CDCl}_{3}\right) \delta$ $2.54(\mathrm{~d}, J=1.0 \mathrm{~Hz}, 3 \mathrm{H}), 3.25,3.31,5.34$ (ABX, $J=17.0,8.0$, $6.8 \mathrm{~Hz}, 3 \mathrm{H}), 4.48(\mathrm{~s}, 2 \mathrm{H}), 5.56(\mathrm{~s}, 2 \mathrm{H}), 6.87$ (br s, $1 \mathrm{H})$, 7.24-7.27 (m, 2H), 7.31-7.37 (m, 3H), $7.41(\mathrm{~d}, J=8.9 \mathrm{~Hz}, 1 \mathrm{H})$, $7.93(\mathrm{~d}, J=8.9 \mathrm{~Hz}, 1 \mathrm{H}) ;{ }^{13} \mathrm{C} \mathrm{NMR}\left(125 \mathrm{MHz}, \mathrm{CDCl}_{3}\right) \delta 12.88$, 22.04, 28.75, 70.20, 71.98, 102.00, 110.78, 115.41, 117.06, 
$118.19,119.09,127.02,127.65,128.31,128.64,136.25,139.80$, 141.77, 142.14; IR (KBr, cm $\left.{ }^{-1}\right)$ v: 2949, 2249, 2225, 1607, 1562, 1519, 1446, 1399, 1337, 1071, 821, 738, 700; EIMS (70 eV) $m / z$ (\% relative intensity): $374(11)[\mathrm{M}]^{+*}, 340(8), 92(14)$, 91 (100), 65 (8); HRMS-EI $(70 \mathrm{eV}, \mathrm{m} / \mathrm{z})$ : $[\mathrm{M}]^{+}$calcd for $\mathrm{C}_{21} \mathrm{H}_{18} \mathrm{~N}_{4} \mathrm{O}_{3}, 374.1379$; found, 374.1385.

3-(1-Benzyloxymethyl-2-methyl-5-nitro-1 H-indol-4-yl)-3cyano- $\mathbf{N}, \mathbf{N}$-dimethylpropionamide (5f). Orange oil; ${ }^{1} \mathrm{H}$ NMR $\left(500 \mathrm{MHz}, \mathrm{CDCl}_{3}\right) \delta 2.52(\mathrm{~s}, 3 \mathrm{H}), 2.97($ br s, $1 \mathrm{H}), 2.96(\mathrm{~s}, 3 \mathrm{H})$ $2.98(\mathrm{~s}, 3 \mathrm{H}), 3.40(\mathrm{dd}, J=16.4,8.8, \mathrm{~Hz}, 1 \mathrm{H}), 4.47(\mathrm{~s}, 2 \mathrm{H}), 5.44$ $(\mathrm{dd}, J=8.8,5.4 \mathrm{~Hz}, 1 \mathrm{H}), 5.54(\mathrm{~s}, 2 \mathrm{H}), 6.81(\mathrm{~m}, 1 \mathrm{H}), 7.25-7.30$ (m, 2H), 7.31-7.37 (m, 4H), $7.83(\mathrm{~d}, J=9.0 \mathrm{~Hz}, 1 \mathrm{H}) ;{ }^{13} \mathrm{C} \mathrm{NMR}$ $\left(100 \mathrm{MHz} \mathrm{CDCl}_{3}\right) \delta 12.83,28.10,35.69,36.93,36.99,70.07$, 71.94, 102.26, 109.66, 118.88, 119.58, 121.67, 127.32, 127.66, $128.26,128.64,136.45,139.39,141.10,142.39,167.99$; IR $\left(\mathrm{KBr}, \mathrm{cm}^{-1}\right)$ v: 2932, 2244, 1651, 1561, 1519, 1400, 1340, 1267, 1241, 1150, 1070, 822, 737, 700; EIMS (70 eV) m/z (\% relative intensity): $420(2)[\mathrm{M}]^{+*}, 375$ (6), 374 (16), 195 (8), 108 (8), 107 (6), 92 (12), 91 (49); HRMS-EI (70 eV, m/z): [M] ${ }^{+}$ calcd for $\mathrm{C}_{23} \mathrm{H}_{24} \mathrm{~N}_{4} \mathrm{O}_{4}, 420.1798$; found, 420.1796 .

2-(1-Benzyloxymethyl-2-methyl-5-nitro-1 H-indol-4-yl)-5phenylpent-4-enenitrile (5g). Yellow crystals; $\mathrm{mp}$ 130-132 ${ }^{\circ} \mathrm{C} ;{ }^{1} \mathrm{H}$ NMR $\left(500 \mathrm{MHz}, \mathrm{CDCl}_{3}\right) \delta 2.52$ (s, 3H), 2.98 (ddd, $J=13.8,9.1,7.5 \mathrm{~Hz}, 1 \mathrm{H}), 3.13$ (ddd, $J=13.8,7.5,6.0$ $\mathrm{Hz}, 1 \mathrm{H}), 5.09$ (dd, $J=9.1,6.0 \mathrm{~Hz}, 1 \mathrm{H}), 6.31$ (ddd, 15.4, 7.5, 7.5 $\mathrm{Hz}, 1 \mathrm{H}), 6.58$ (d, $J=15.4 \mathrm{~Hz}, 1 \mathrm{H}), 6.90$ (s, 1H), 7.21-7.40 (m, $11 \mathrm{H}), 7.85(\mathrm{~d}, J=8.8 \mathrm{~Hz}, 1 \mathrm{H}) ;{ }^{13} \mathrm{C} \mathrm{NMR}\left(100 \mathrm{MHz}, \mathrm{CDCl}_{3}\right) \delta$ $12.82,32.17,37.05,70.06,71.94,102.88,109.67,118.86$, $119.73,121.77,124.03,126.43,126.92,127.70,127.73,128.29$, $128.55,129.45,134.43,136.44,136.64,139.54,140.92$, 142.10; IR (KBr, cm $\left.{ }^{-1}\right)$ v: 3026, 2239, 2206, 1605, 1556, 1512, 1437, 1400, 1335, 1080, 1070, 967, 817, 764, 742, 734, 693; EIMS (70 eV) $m / z\left(\%\right.$ relative intensity): $451(9)[\mathrm{M}]^{+}, 434(4)$, 405 (4), 334 (15), 117 (22), 115 (13), 105 (6), 91 (100); HRMS-EI $(70 \mathrm{eV}, \mathrm{m} / \mathrm{z}):[\mathrm{M}]^{+}$calcd for $\mathrm{C}_{28} \mathrm{H}_{25} \mathrm{~N}_{3} \mathrm{O}_{3}$, 451.1896; found, 451.1890 .

\section{Cyclization of 5-nitroindol-4-ylacetonitriles to pyrrolo[3,2-e]indoles - Typical procedure}

To a solution of indole derivative $5(1 \mathrm{mmol})$ and DBU $(0.75 \mathrm{~g}$, $5 \mathrm{mmol})$ in DMF $(5 \mathrm{~mL}) \mathrm{Me}_{3} \mathrm{SiCl}(0.54 \mathrm{~g}, 5 \mathrm{mmol})$ was added at room temperature. The reaction mixture was stirred for 20-30 min (TLC control), quenched with diluted $\mathrm{HCl}$, extracted with EtOAc $(3 \times 30 \mathrm{~mL})$ and dried with $\mathrm{Na}_{2} \mathrm{SO}_{4}$. After evaporation of the solvent the residue was purified by column chromatography $\left(\mathrm{SiO}_{2}\right.$, hexane-EtOAc, 2:1).

6-Benzyloxymethyl-1-cyano-3-hydroxy-7-methyl-3,6-dihydropyrrolo[3,2-e]indole-2-carboxylic acid ethyl ester (6a).
Yellow crystals; mp $119-121{ }^{\circ} \mathrm{C} ;{ }^{1} \mathrm{H}$ NMR $(500 \mathrm{MHz}$, DMSO$\left.d_{6}\right) \delta 1.37(\mathrm{t}, J=7.1 \mathrm{~Hz}, 3 \mathrm{H}), 2.58(\mathrm{~s}, 3 \mathrm{H}), 4.41(\mathrm{q}, J=7.1 \mathrm{~Hz}$, $2 \mathrm{H}), 4.48(\mathrm{~s}, 2 \mathrm{H}), 5.77(\mathrm{~s}, 2 \mathrm{H}), 6.84(\mathrm{~m}, 1 \mathrm{H}), 7.23-7.36(\mathrm{~m}$, $5 \mathrm{H}), 7.73(\mathrm{~d}, J=9.1 \mathrm{~Hz}, 1 \mathrm{H}), 11.22(\mathrm{br} \mathrm{s}, 1 \mathrm{H}) ;{ }^{13} \mathrm{C} \mathrm{NMR}(125$ $\left.\mathrm{MHz}, \mathrm{CD}_{3} \mathrm{COCD}_{3}\right) \delta 12.64,14.44,62.30,70.32,73.18,85.95$, 100.46, 104.26, 112.46, 116.13, 116.59, 119.80, 126.68, 128.37, $128.41,128.45,129.12,131.11,134.06,138.27,138.73,159.36$; IR $\left(\mathrm{KBr}, \mathrm{cm}^{-1}\right)$ v: 2987, 2212, 1753, 1682, 1618, 1597, 1499, 1453, 1430, 1368, 1333, 1321, 1265, 1136, 1062, 1028, 775; EIMS $(70 \mathrm{eV}) \mathrm{m} / \mathrm{z}$ (\% relative intensity): $403(38)[\mathrm{M}]^{+\cdot}, 387$ (13), 311 (11), 297 (14), 283 (13), 281 (6), 192 (5), 92 (8), 91 (100); HRMS-EI $(70 \mathrm{eV}, \mathrm{m} / \mathrm{z}):[\mathrm{M}]^{+}$calcd for $\mathrm{C}_{23} \mathrm{H}_{21} \mathrm{~N}_{3} \mathrm{O}_{4}$, 403.1532; found, 403.1519 .

2-Acetyl-6-benzyloxymethyl-3-hydroxy-7-methyl-3,6-dihydropyrrolo $[3,2-e]$ indole-1-carbonitrile $(6 \mathrm{~b})$. Yellow crystals; mp 194-196 ${ }^{\circ} \mathrm{C} ;{ }^{1} \mathrm{H}$ NMR (500 MHz, DMSO- $\left.d_{6}\right) \delta 2.53$ $(\mathrm{d}, J=0.7 \mathrm{~Hz}, 3 \mathrm{H}), 2.74(\mathrm{~s}, 3 \mathrm{H}), 4.85(\mathrm{~s}, 2 \mathrm{H}), 5.74(\mathrm{~s}, 2 \mathrm{H}), 6.75$ (br s, 1H), 7.24-7.29 (m, 3H), 7.30-7.34 (m, 2H), 7.35 (d, $J=$ $9.1 \mathrm{~Hz}, 1 \mathrm{H}), 7.76(\mathrm{~d}, J=9.1 \mathrm{~Hz}, 1 \mathrm{H}), 12.49$ (br s, 1H); ${ }^{13} \mathrm{C}$ NMR $\left(125 \mathrm{MHz}, \mathrm{DMSO}-d_{6}\right) \delta 12.23,29.97,69.15,72.20$, 82.41, 99.30, 103.81, 112.04, 115.60, 116.54, 118.09, 127.44, $127.59,128.27,129.90,132.66,134.36,137.32,137.53$, 187.55; IR (KBr, cm $\left.{ }^{-1}\right)$ v: 2917, 2216, 1645, 1594, 1545, 1510, 1483, 1444, 1426, 1366, 1314, 1236, 1121, 1088, 1020, 990; EIMS (70 eV) $m / z$ (\% relative intensity): $373(9)[\mathrm{M}]^{+\cdot}, 358$ (9), 357 (39), 327 (15), 326 (5), 252 (6), 251 (23), 250 (8), 237 (6), 236 (7), 208 (8), 194 (7), 108 (5), 106 (5), 92 (8), 91 (100); HRMS-EI $(70 \mathrm{eV}, \mathrm{m} / \mathrm{z}):[\mathrm{M}]^{+}$calcd for $\mathrm{C}_{22} \mathrm{H}_{19} \mathrm{~N}_{3} \mathrm{O}_{3}$, 373.1426; found, 373.1421.

6-Benzyloxymethyl-2-(2,2-dimethylpropionyl)-3-hydroxy-7methyl-3,6-dihydropyrrolo[3,2-e] indole-1-carbonitrile (6c). Brown solid; mp $155-157{ }^{\circ} \mathrm{C} ;{ }^{1} \mathrm{H}$ NMR (500 MHz, DMSO- $d_{6}$ ) $\delta 1.32(\mathrm{~s}, 9 \mathrm{H}), 2.52(\mathrm{~d}, J=0.8 \mathrm{~Hz}, 3 \mathrm{H}), 4.47(\mathrm{~s}, 2 \mathrm{H}), 5.73(\mathrm{~s}$, 2H), 6.68 (br s, 1H), 7.24-7.35 (m, 6H), 7.67 (d, $J=9.0 \mathrm{~Hz}$, $1 \mathrm{H}), 12.50$ (br s, $1 \mathrm{H}) ;{ }^{13} \mathrm{C} \mathrm{NMR}\left(125 \mathrm{MHz}, \mathrm{DMSO}-d_{6}\right) \delta 12.27$, 26.00, 44.68, 69.08, 72.21, 78.41, 98.67, 103.64, 109.66, $114.83,116.34,118.20,127.43,127.59,128.00,128.28,132.87$, 137.25, 137.30, 137.58, 202.17; IR $\left(\mathrm{KBr}, \mathrm{cm}^{-1}\right)$ v: 2969, 2244, 1707, 1562, 1519, 1477, 1398, 1340, 1071, 1029; EIMS (70 eV) $\mathrm{m} / \mathrm{z}$ (\% relative intensity): $415(9)[\mathrm{M}]^{+}, 400$ (15), 399 (59), 369 (6), 342 (7), 313 (5), 312 (17), 294 (5), 293 (19), 292 (8), 285 (7), 284 (8), 236 (8), 208 (5), 194 (6), 193 (5), 108 (6), 92 (8), 91 (100); HRMS-EI $(70 \mathrm{eV}, \mathrm{m} / z):[\mathrm{M}]^{+}$calcd for $\mathrm{C}_{25} \mathrm{H}_{25} \mathrm{~N}_{3} \mathrm{O}_{3}, 415.1896$; found, 415.1878 .

2-Benzoyl-6-benzyloxymethyl-7-methyl-3-hydroxy-3,6-dihydropyrrolo[3,2-e] indole-1-carbonitrile (6d). Red crystals; mp $130-132{ }^{\circ} \mathrm{C} ;{ }^{1} \mathrm{H}$ NMR $\left(500 \mathrm{MHz}, \mathrm{CDCl}_{3}\right) \delta 2.51(\mathrm{~s}, 3 \mathrm{H})$, $4.44(\mathrm{~s}, 2 \mathrm{H}), 5.60(\mathrm{~s}, 2 \mathrm{H}), 6.93(\mathrm{~s}, 1 \mathrm{H}), 7.24-7.28(\mathrm{~m}, 2 \mathrm{H})$, 
$7.29-7.39(\mathrm{~m}, 4 \mathrm{H}), 7.53(\mathrm{~d}, J=9.1 \mathrm{~Hz}, 1 \mathrm{H}), 7.58-7.63(\mathrm{~m}, 2 \mathrm{H})$, 7.69-7.75 (m, 1H), 7.97-8.02 (m, 2H), 12.56 (br s, 1H); ${ }^{13} \mathrm{C}$ NMR $\left(125 \mathrm{MHz}, \mathrm{CDCl}_{3}\right) \delta 12.67,69.59,72.04,85.65$, 101.26, 103.34, 113.27, 115.64, 117.69, 119.00, 126.39, 127.74, 128.08, 128.56, 128.69, 128.87, 129.95, 132.94, 134.15, 135.87, 136.85, 137.15, 189.1; IR (KBr, cm $\left.{ }^{-1}\right)$ v: 2921, 2215, 1639, 1599, 1569, 1496, 1479, 1424, 1367, 1329, 1314, 1260, 1085, 1072, 778, 732, 693; ESIMS (MeOH) $m / z: 436[\mathrm{M}+\mathrm{H}]^{+}, 458$ $[\mathrm{M}+\mathrm{Na}]^{+}$; HRMS-EI $(70 \mathrm{eV}, \mathrm{m} / z):[\mathrm{M}+\mathrm{Na}]^{+}$calcd for $\mathrm{C}_{27} \mathrm{H}_{21} \mathrm{~N}_{3} \mathrm{O}_{3} \mathrm{Na}$, 458.1475; found, 458.1495 .

6-Benzyloxymethyl-3-hydroxy-7-methyl-3,6-dihydropyrrolo[3,2-e]indole-1,2-dicarbonitrile (6e). Brownish crystals; mp 150-152 ${ }^{\circ} \mathrm{C} ;{ }^{1} \mathrm{H}$ NMR $\left(500 \mathrm{MHz}, \mathrm{DMSO}-d_{6}\right) \delta 2.53$ (s, $3 \mathrm{H}), 4.47$ (s, 2H), 5.75 (s, 2H), $6.70(\mathrm{br} \mathrm{s}, 1 \mathrm{H}), 7.22-7.34(\mathrm{~m}$, $5 \mathrm{H}), 7.36(\mathrm{~d}, J=9.1 \mathrm{~Hz}, 1 \mathrm{H}), 7.83(\mathrm{~d}, J=9.1 \mathrm{~Hz}, 1 \mathrm{H}), 13.29$ (br s, $1 \mathrm{H}) ;{ }^{13} \mathrm{C}$ NMR (125 MHz, DMSO- $\left.d_{6}\right) \delta 12.26,69.22$, 72.30, 99.16, 103.44, 108.90, 110.30, 112.65, 114.14, 115.21, 117.70, 127.47, 127.64, 128.31, 128.33, 129.46, 132.86, 137.52, 138.01; IR (KBr, cm $\left.{ }^{-1}\right)$ v: 2922, 2224, 1544, 1484, 1393, 1351, 1319, 1209, 1145, 1064, 1024, 774, 753, 699; ESIMS (MeOH/ $\left.\mathrm{CH}_{2} \mathrm{Cl}_{2}\right) \mathrm{m} / z: 357[\mathrm{M}+\mathrm{H}]^{+}, 379[\mathrm{M}+\mathrm{Na}]^{+}, 735[2 \mathrm{M}+\mathrm{Na}]^{+}$.

6-Benzyloxymethyl-1-cyano-3-hydroxy-7-methyl-3,6-dihydropyrrolo[3,2-e]indole-2-carboxylic acid dimethylamide (6f). Brown semisolid; ${ }^{1} \mathrm{H}$ NMR (500 MHz, DMSO- $\left.d_{6}\right) \delta 2.49$ (s, 3H), 3.02 (s, 3H), 3.30 (br s, 3H), 4.44 (s, 2H), $5.68(\mathrm{~s}, 2 \mathrm{H})$, 6.61 (br s, 1H), 7.22-7.33 (m, 6H), $7.52(\mathrm{~d}, J=9.0 \mathrm{~Hz}, 1 \mathrm{H})$, 13.24 (br s, $1 \mathrm{H}) ;{ }^{13} \mathrm{C}$ NMR (125 MHz, DMSO-d 6 ) $\delta 12.21$, $34.38,37.24,68.92,72.06,98.73,104.84,107.15,115.29$, 117.24, 117.99, 127.35, 127.49, 127.93, 128.22, 132.58, 135.89, 137.64 (3 peaks not visible); IR $\left(\mathrm{KBr}, \mathrm{cm}^{-1}\right)$ v: 2930, 2208, $1615,1525,1445,1390,1363,1319,1258,1209,1121,1066$, 778, 738, 698; EIMS (70 eV) $m / z$ (\% relative intensity): 402 (10) $[\mathrm{M}]^{+\cdot}, 387$ (7), 386 (27), 312 (6), 311 (17), 280 (5), 265 (6), 221 (5), 220 (6), 213 (6), 193 (6), 192 (5), 165 (14), 135 (7), 108 (39), 107 (10), 105 (7), 92 (10), 91 (100); HRMS-EI (70 eV, m/z): $[\mathrm{M}]^{+}$calcd for $\mathrm{C}_{23} \mathrm{H}_{22} \mathrm{~N}_{4} \mathrm{O}_{3}, 402.1692$; found, 402.1684 .

6-Benzyloxymethyl-3-hydroxy-7-methyl-2-((E)-styryl)-3,6dihydropyrrolo $[3,2-e]$ indole-1-carbonitrile $(6 \mathrm{~g})$. Dark green solid; mp $130-132{ }^{\circ} \mathrm{C} ;{ }^{1} \mathrm{H}$ NMR (500 MHz, DMSO- $\left.d_{6}\right) \delta 2.52$ (d, $J=0.8 \mathrm{~Hz}, 3 \mathrm{H}), 4.48(\mathrm{~s}, 2 \mathrm{H}), 5.71(\mathrm{~s}, 2 \mathrm{H}), 6.69$ (br s, 1H), $7.21-7.40(\mathrm{~m}, 8 \mathrm{H}), 7.44-7.48(\mathrm{~m}, 2 \mathrm{H}), 7.56(\mathrm{~d}, J=9.0 \mathrm{~Hz}, 1 \mathrm{H})$, $7.69(\mathrm{~m}, 2 \mathrm{H}), 7.77(\mathrm{~d}, J=16.5 \mathrm{~Hz}, 1 \mathrm{H}), 12.10(\mathrm{~s}, 1 \mathrm{H})$; ${ }^{13} \mathrm{C}$ NMR (125 MHz, DMSO- $d_{6}$ ) $\delta 12.26,69.07,72.18,98.74$, $103.21,108.35,113.73,115.56,117.83,117.85,126.83,127.42$, 127.56, 127.66, 128.27, 128.60, 128.91, 129.05, 132.84, 133.04, 135.95, 136.87, 136.99, 137.63; IR (KBr, cm $\left.{ }^{-1}\right)$ v: 2923, 2200, $1739,1547,1467,1389,1361,1339,1317,1203,1142,1066$,
955, 746; EIMS (70 eV) $\mathrm{m} / z$ (\% relative intensity): 433 (2) $[\mathrm{M}]^{+\cdot}, 431$ (16), 325 (10), 297 (11), 165 (28), 108 (92), 107 (68), 106 (35), 105 (46), 92 (8), 91 (82); HRMS-ESI (m/z): [M $+\mathrm{Na}]^{+}$calcd for $\mathrm{C}_{28} \mathrm{H}_{23} \mathrm{~N}_{3} \mathrm{O}_{2} \mathrm{Na}, 456.1688$; found, 456.1685 .

6 - B e nzy lox y met hy l- 1 - c y a n o - 7 - meth y l-3, 6 dihydropyrrolo[3,2-e]indole-2-carboxylic acid ethyl ester (8a). Pale creamy crystals; mp 215-217 ${ }^{\circ} \mathrm{C} ;{ }^{1} \mathrm{H}$ NMR $(500$ MHz, DMSO- $\left.d_{6}\right) \delta 1.40(\mathrm{t}, J=7.1 \mathrm{~Hz}, 3 \mathrm{H}), 2.52(\mathrm{~s}, 3 \mathrm{H}), 4.44$ (q, $J=7.1 \mathrm{~Hz}, 2 \mathrm{H}), 4.47(\mathrm{~s}, 2 \mathrm{H}), 5.71(\mathrm{~s}, 2 \mathrm{H}), 6.74(\mathrm{br} \mathrm{s}, 1 \mathrm{H})$, 7.24-7.35 (m, 6H), 12.99 (br s, $1 \mathrm{H}) ;{ }^{13} \mathrm{C}$ NMR (125 MHz, DMSO- $\left.d_{6}\right) \delta 12.23,14.07,61.35,69.09,72.18,87.59,99.08$, 106.70, 111.12, 115.93, 118.34, 119.90, 127.42, 127.57, 128.27, 129.34, 131.58, 132.28, 136.77, 137.57, 159.10; IR (KBr, cm $\left.{ }^{-1}\right)$ v: $3264,2219,1689,1527,1455,1434,1362,1315,1261,1064$, 1055, 1025, 743; EIMS (70 eV) $\mathrm{m} / z$ (\% relative intensity): 387 (50) $[\mathrm{M}]^{+\cdot}, 312$ (8), 311 (37), 281 (14), 280 (5), 266 (5), 235 (6), 220 (12), 206 (5), 192 (6), 165 (5), 92 (8), 91 (100); HRMS-EI $(m / z)$ : $[\mathrm{M}]^{+}$calcd for $\mathrm{C}_{23} \mathrm{H}_{21} \mathrm{~N}_{3} \mathrm{O}_{3}, 387.1583$; found, 387.1587 .

2 - B enzoyl-6 - be nzyloxymethy l- 7 - methyl-3,6dihydropyrrolo[3,2-e]indole-1-carbonitrile (8d). Yellow crystals; mp $180-182{ }^{\circ} \mathrm{C} ;{ }^{1} \mathrm{H}$ NMR (500 MHz, DMSO-d $\left.{ }_{6}\right) \delta$ $2.53(\mathrm{~s}, 3 \mathrm{H}), 4.47(\mathrm{~s}, 2 \mathrm{H}), 5.74(\mathrm{~s}, 2 \mathrm{H}), 6.75(\mathrm{~s}, 1 \mathrm{H}), 7.23-7.38$ (m, 6H), 7.60-7.67 (m, 2H), 7.69-7.78 (m, 2H), 7.89-7.94 (m, 2H), 12.93 (br s, $1 \mathrm{H}) ;{ }^{13} \mathrm{C}$ NMR (125 MHz, DMSO-d $\left.d_{6}\right) \delta 12.24$, 69.07, 72.17, 88.59, 99.28, 106.82, 111.83, 116.11, 118.42, $120.51,127.44,127.59,128.28,128.67,129.38,132.20,132.38$, $133.11,136.36,136.86,136.97,137.57,185.27$; IR $\left(\mathrm{KBr}, \mathrm{cm}^{-1}\right)$ v: 3265, 2217, 1710, 1624, 1540, 1510, 1408, 1329, 1254, 1062, 942, 739, 696; ESIMS (MeOH) $m / z: 420[\mathrm{M}+\mathrm{H}]^{+}, 442[\mathrm{M}+$ $\mathrm{Na}]^{+}, 861[2 \mathrm{M}+\mathrm{Na}]^{+}$; HRMS-ESI $(m / z):[\mathrm{M}+\mathrm{Na}]^{+}$calcd for $\mathrm{C}_{27} \mathrm{H}_{21} \mathrm{~N}_{3} \mathrm{O}_{2} \mathrm{Na}$, 442.1526; found, 442.1544 .

\section{Debenzyloxymethylation of compound $\mathbf{8 a}$}

Compound 8 (100 mg, $0.26 \mathrm{mmol})$, ammonium formate $(0.16 \mathrm{~g}$, $10 \mathrm{mmol})$ and $10 \%$ palladium on charcoal $(90 \mathrm{mg})$ were suspended in isopropanol $(5 \mathrm{~mL})$, flushed with argon for $5 \mathrm{~min}$ and then heated in a sealed tube at $95{ }^{\circ} \mathrm{C}$ overnight. Then the reaction mixture was passed through Celite, washed with dichloromethane-methanol, 1:1 (15 mL). After evaporation the residue was purified by column chromatography on silica gel with hexane-ethyl acetate (gradient 4:1 to 1:1). The following compounds were obtained:

1-Cyano-7-methyl-3,6-dihydropyrrolo[3,2-e]indole-2carboxylic acid ethyl ester (9a). Yield $22 \%$; $\mathrm{mp}>280{ }^{\circ} \mathrm{C}$; ${ }^{1} \mathrm{H}$ NMR (500 MHz, DMSO-d $) \delta 1.39(\mathrm{t}, J=7.1 \mathrm{~Hz}, 3 \mathrm{H}), 2.45$ $(\mathrm{s}, 3 \mathrm{H}), 4.42(\mathrm{q}, J=7.1 \mathrm{~Hz}, 2 \mathrm{H}), 6.56(\mathrm{~s}, 1 \mathrm{H}), 7.19(\mathrm{~d}, J=8.8$ $\mathrm{Hz}, 1 \mathrm{H}), 7.40$ (d, $J=8.8 \mathrm{~Hz}, 1 \mathrm{H}), 11.33$ (s, 1H), 12.85 (s, 1H); 
${ }^{13} \mathrm{C}$ NMR (125 MHz, DMSO- $d_{6}$ ) $\delta 13.27,14.07,61.19,87.56$, 97.30, 105.78, 112.34, 116.06, 118.75, 120.01, 128.49, 131.03, 131.09, 135.00, 159.14; IR (film, $\mathrm{cm}^{-1}$ ) v: 3265, 2256, 1690, 1549, 1526, 1439, 1363, 1338, 1254, 1115, 1073,1016; EIMS (70 eV) $\mathrm{m} / \mathrm{z}$ (\% relative intensity): 267 (75) $[\mathrm{M}]^{+*}, 222$ (26), 221 (100), 194 (25), 193 (55), 167 (17).

1,7-Dimethyl-3,6-dihydropyrrolo[3,2-e] indole-2-carboxylic acid ethyl ester (10a). Yield 34\%; mp 225-227 ${ }^{\circ} \mathrm{C}$ dec; ${ }^{1} \mathrm{H}$ NMR (500 MHz, DMSO- $\left.d_{6}\right) \delta 1.35(\mathrm{t}, J=7.0 \mathrm{~Hz}, 3 \mathrm{H}), 2.42$ (s, 3H), $2.72(\mathrm{~s}, 3 \mathrm{H}), 4.32(\mathrm{q}, J=7.0 \mathrm{~Hz}, 2 \mathrm{H}), 6.46(\mathrm{~s}, 1 \mathrm{H}), 7.04$ $(\mathrm{d}, J=8.8 \mathrm{~Hz}, 1 \mathrm{H}), 7.23(\mathrm{~d}, J=8.8 \mathrm{~Hz}, 1 \mathrm{H}), 10.97(\mathrm{~s}, 1 \mathrm{H})$, $11.21(\mathrm{~s}, 1 \mathrm{H}) ;{ }^{13} \mathrm{C}$ NMR (125 MHz, DMSO- $\left.d_{6}\right) \delta 11.57,13.35$, 14.40, 59.47, 98.24, 105.38, 110.99, 118.48, 119.62, 120.46, $120.54,129.65,131.77,133.23,162.01$; IR $\left(\mathrm{KBr}, \mathrm{cm}^{-1}\right)$ v: $3318,1675,1548,1534,1437,1362,1334,1291,1243,1209$, 1190, 1117, 1092, 1017, 772 .

\section{Supporting Information}

\section{Supporting Information File 1}

${ }^{1} \mathrm{H}$ and ${ }^{13} \mathrm{C}$ NMR, IR and mass spectra for compounds 4 ,

5a-g, 6a-g, 8a, 8d, 9a and 10a.

[http://www.beilstein-journals.org/bjoc/content/ supplementary/1860-5397-9-107-S1.pdf]

\section{Acknowledgements}

This work was supported by the National Scientific Center, Grant NN 204193038.

\section{References}

1. Ghosh, N.; Sheldrake, H. M.; Searcey, M.; Pors, K. Curr. Top. Med. Chem. 2009, 9, 1494-1524. doi:10.2174/156802609789909812

2. Tichenor, M. S.; Boger, D. L. Nat. Prod. Rep. 2008, 25, 220-226. doi:10.1039/b705665f

3. Samsoniya, Sh. A.; Kadzhrishvili, D. O.; Chikvaidze, I. Sh. Pharm. Chem. J. 2011, 45, 22-25. doi:10.1007/s11094-011-0553-7

4. Okano, K.; Mitsuhashi, N.; Tokuyama, H. Chem. Commun. 2010, 46, 2641-2643. doi:10.1039/b926965g

5. Rawal, V. H.; Jones, R. J.; Cava, M. P. J. Org. Chem. 1987, 52, 19-28. doi:10.1021/jo00377a004

6. Macor, J. E.; Forman, J. T.; Post, R. J.; Ryan, K. Tetrahedron Lett. 1997, 38, 1673-1676. doi:10.1016/S0040-4039(97)00198-6

7. Mąkosza, M. Synthesis 2011, 2341-2356. doi:10.1055/s-0030-1260668

8. Mąkosza, M.; Wojciechowski, K. Nucleophilic Substitution of Hydrogen - an Efficient Tool in Synthesis of Heterocyclic Compounds. In Targets in Heterocyclic Systems: Chemistry and Properties; Attanasi, O., Ed.; Soc. Chimica Italiana: Rome, 2011; Vol. 14, pp 19-48.

9. Mąkosza, M. Chem. Soc. Rev. 2010, 39, 2855-2868. doi:10.1039/b822559c
10. Mąkosza, M.; Wojciechowski, K. Chem. Rev. 2004, 104, 2631-2666. doi:10.1021/cr020086+

11. Mąkosza, M.; Wojciechowski, K. Heterocycles 2001, 54, 445-474. doi:10.3987/REV-00-SR(I)2

12. Wojciechowski, K.; Mąkosza, M. Synthesis 1989, 106-109. doi:10.1055/s-1989-34082

13. Wróbel, Z.; Makkosza, M. Synlett 1993, 597-598. doi:10.1055/s-1993-22544

14. Wróbel, Z.; Mąkosza, M. Tetrahedron 1993, 49, 5315-5326. doi:10.1016/S0040-4020(01)82380-2

15. Wróbel, Z.; Mąkosza, M. Tetrahedron 1997, 53, 5501-5514. doi:10.1016/S0040-4020(97)00208-1

16. Wróbel, Z.; Wojciechowski, K.; Kwast, A.; Gajda, N. Synlett 2010, 2435-2438. doi:10.1055/s-0030-1258555

17. Bujok, R.; Trawczyński, A.; Wróbel, Z.; Wojciechowski, K. Synlett 2012 , 2682-2686. doi:10.1055/s-0032-1317380

18. Bujok, R.; Wróbel, Z.; Wojciechowski, K. Synlett 2012, 1315-1320. doi:10.1055/s-0031-1291044

19. Brown, G. R.; Foubister, A. J. Synthesis 1982, 1036-1037. doi:10.1055/s-1982-30054

\section{License and Terms}

This is an Open Access article under the terms of the Creative Commons Attribution License (http://creativecommons.org/licenses/by/2.0), which permits unrestricted use, distribution, and reproduction in any medium, provided the original work is properly cited.

The license is subject to the Beilstein Journal of Organic Chemistry terms and conditions:

(http://www.beilstein-journals.org/bjoc)

The definitive version of this article is the electronic one which can be found at: doi:10.3762/bjoc.9.107 